\title{
Frequent Allelic Imbalance and Loss of Protein Expression of the DNA Repair Gene hOGG1 in Head and Neck Squamous Cell Carcinoma
}

\author{
Chun-Yang Fan, Ke La Liu, Huai Yun Huang, E. Leon Barnes, Patricia A. Swalsky, \\ Anke Bakker, Jeffrey Woods, and Sydney D. Finkelstein
}

Department of Pathology (C-YF, KLL, HYH), University of Arkansas for Medical Sciences, Little Rock, Arkansas, and University of Pittsburgh Medical Center (ELB, PAS, AB, JW, SDF), Pittsburgh, Pennsylvania

\begin{abstract}
SUMMARY: Reactive oxygen species produced by aerobic cellular metabolism or through exposure to environmental carcinogens can cause oxidative DNA damage by generating DNA base lesions and strand breakage. Prime among these base lesions is the conversion of guanine to 8-oxoguanine. Among 20 or so oxidative DNA base lesions, 8-oxoguanine is the most abundant and is critical in terms of mutagenesis because it is capable of mispairing with adenine, which, if not sufficiently repaired, may lead to G:C to T:A transversion upon DNA replication. The gene encoding human 8-oxoguanine DNA glycosylase 1 (hOGG1), capable of excision repair of 8-oxoguanine, has been recently cloned, characterized, and mapped to the short arm of chromosome 3 (3p25-26), a region showing frequent loss of heterozygosity (LOH) in head and neck squamous cell carcinoma (HNSCC). In the present study, we developed a tissue microdissection approach designed for use with formalin-fixed, paraffin-embedded specimens which is capable of detecting and characterizing the hOGG1 allelic loss using two highly informative, intragenic single nucleotide polymorphisms. Among 45 cases of HNSCC, 18 cases were informative. We analyzed these 18 cases and found that 11 showed evidence of hOGG1 allelic loss. By immunohistochemical staining on a total of 71 HNSCC cases using a commercially available anti-hOGG1 antibody, we showed that hOGG1 gene expression was markedly suppressed in up to $38 \%$ of the cases. The frequent allelic imbalance and suppression of the hOGG1 gene thus imply that repair for oxidative DNA damages may be relevant in future studies on head and neck squamous carcinogenesis. (Lab Invest 2001, 81:1429-1438).
\end{abstract}

H ead and neck tumors are a heterogeneous 1 group of neoplasms that display a wide range of biologic behaviors. In the United States, excluding those from skin, central nervous system, eye, thyroid and lymph nodes, tumors of the head and neck account for $5 \%$ of the total cancer burden.

Of all head and neck tumors, $80 \%$ to $90 \%$ are squamous cell carcinoma (SCC). The strong association with tobacco and alcohol use makes head and neck squamous cell carcinoma (HNSCC) one of the most preventable malignant diseases (Blot et al, 1988; Franceschi et al, 1990). Basic research on HNSCC has been largely neglected because of the tumor's rarity. However, there are approximately 50,000 new cases of HNSCC in the United States annually (Vokes et al, 1993) and the incidence is expected to climb as a result of the increasing number of female and adolescent smokers (Centers for Disease Control, 1990). In addition, despite the great emphasis on early detec-

Received June 21, 2001.

This work was supported in part by research funds from the Department of Pathology, University of Pittsburgh Medical Center and from the Department of Pathology and Otolaryngology, University of Arkansas for Medical Sciences.

Address reprint requests to: Dr. Sydney D. Finkelstein, Department of Pathology, University of Pittsburgh Medical Center, 200 Lothrop Street, Pittsburgh, PA 15213. E-mail: finkelsteinsd@msx.upmc.edu tion and the efforts to improve multimodality treatment management, 5-year survival rate for HNSCC patients does not exceed 50\% (Ries et al, 1990). The pressing epidemiologic problem and the lack of effective management for patients with HNSCC thus strengthen the need for more extensive research on HNSCC at the molecular and genetic levels.

Previous studies have identified a number of genetic abnormalities in HNSCC, including nonrandom cytogenetic aberrations (Cowan et al, 1992; Heo et al, 1989), mutation or overexpression of the $\mathrm{H}$-ras gene (Saranath et al, 1991) and p53 gene (Brennan et al, 1995; Field et al, 1992), and overexpression of epidermal growth factor receptor (Grandis et al, 1998a, 1998b). Inactivation of p16 gene (CDKN2), a G1 cyclindependent kinase inhibitor and DCC (deletion in colon cancer) gene, has also been reported (Reed et al, 1996).

A high frequency of chromosomal aberrations involving chromosome $3 p$ has been reported in HNSCC (Cowan et al, 1992; Heo et al, 1989), small-cell lung cancer (Whang-Peng et al, 1982), and esophageal carcinomas (Whang-Peng et al, 1990) by cytogenetic analysis. The observation of similar genetic changes in these three tumors and their common association with tobacco use (Franceschi et al, 1990) strengthen the hypothesis that HNSCC may share similar mecha- 
nisms of carcinogenesis with lung and esophageal cancers.

Three discrete regions of deletion at chromosome $3 p$ in HNSCC have been identified (Maestro et al, 1993; Wu et al, 1994), suggesting the presence of potential tumor suppressor loci. However, the identities of these presumptive tumor suppressor genes remain elusive.

Free radicals, which are found in normal aerobic cellular metabolism, are molecules with one or more unpaired electrons as a result of exposure to ionizing radiation or to environmental chemical carcinogens (Dizdaroglu, 1991). Electron acceptors such as molecular oxygen react easily with free radicals to become reactive oxygen species (ROS), among them are superoxide radical, hydrogen peroxide $\left(\mathrm{H}_{2} \mathrm{O}_{2}\right)$ and hydroxyl radical $\left({ }^{\circ} \mathrm{OH}\right)$. ROS are highly reactive and can directly attack DNA, generating oxidative DNA base lesions and strand breakage (Dizdaroglu, 1991). Among 20 or so such DNA base lesions, produced by ROS, 8-oxoguanine is the most abundant (Gajewski et al, 1990) and, if not sufficiently repaired, can cause potential mutation by mispairing with adenine, yielding $\mathrm{G}: \mathrm{C}$ to $\mathrm{T}: \mathrm{A}$ transversion on DNA replication (Cheng et al, 1992).

Genes responsible for repair of oxidative DNA damage have been isolated and studied in great detail in Escherichia coli. Three such members, MutM, MutT, and MutY, possess the capacity to repair 8-oxoguanine-induced mutation should it arise in the bacterial genome (Boiteux et al, 1992; Michaels et al, 1992). A closely related yeast gene, 8-oxoguanine DNA glycosylase (yOGG1), has been isolated in Saccharomyces cerevisiae (Van der Kemp et al, 1996). The human homolog of this gene, human 8-oxoguanine DNA glycosylase 1 (hOGG1), has recently been cloned and characterized (Radicella et al, 1997). The complete genomic sequence of hOGG1 has been reported including the finding of several intragenic single nucleotide polymorphism (SNP) sites (Kohno et al, 1998).

The hOGG1 gene is located at 3p25-26, a region frequently deleted in HNSCC (Maestro et al, 1993; Wu et al, 1994) as well as in lung cancer (Hibi et al, 1992). Loss of heterozygosity (LOH) or mutations in the hOGG1 gene was previously reported in human lung (Chevillard et al, 1998; Kohno et al, 1998), kidney (Chevillard et al, 1998), and gastric (Shinmura et al, 1998) cancers. However, direct DNA sequencing after single strand conformation polymorphism (SSCP) analysis and PCR amplification of the hOGG1 gene transcripts detected only rare mutations in these tumors (Chevillard et al, 1998; Kohno et al, 1998; Shinmura et al, 1998).

To widen the scope of DNA repair dysfunction analysis, we searched for hOGG1 allelic loss in a series of patients with oral and laryngeal squamous cell carcinoma. Using genomic information currently available, we developed a tissue microdissection approach for detection and characterization of allelic loss based on two highly informative intragenic single nucleotide polymorphism (SNP) sites (Kohno et al, 1998). Our findings reported here indicate that hOGG1 gene allelic loss is very frequent, being detected in 11 of $18(61.1 \%)$ cases. By IHC staining procedure, we also found that hOGG1 gene expression was suppressed in 35 of 71 (49\%) cases. These data suggest that hOGG1 gene function may be relevant in the head and neck squamous carcinogenesis.

\section{Results}

\section{LOH of the hOGG1 Gene}

Tissue for hOGG1 genotyping was collected by microdissection from unstained histologic sections of 45 HNSCC cases. After removal of representative normal minor salivary gland, benign hyperplastic squamous mucosa, squamous carcinoma in situ, and invasive SCC, postmicrodissected tissue sections were stained to evaluated accuracy of sample removal (Fig. 1). Whereas inclusion of nonneoplastic cellular elements could not be totally excluded, their contribution to the overall tumor sample for genotyping was judged to be small, amounting to less than $10 \%$ total sample volume (Fig. 1H).

SNP sites at two intragenic sites of hOGG1 were used to identify informative patients for LOH determination (Fig. 2). Sixteen of 45 (36\%) patients were found to be heterozygous for the intron 4 SNP (Tables 1 and 2). Remaining noninformative cases for the intron 4 SNP site consisted of 19 homozygous cytosine and 10 homozygous guanine alleles. The exon 7 SNP was found to be in linkage disequilibrium with the intron 4 SNP, with most but not all subjects found to be informative for both SNP sites (Tables 1 and 2). The noninformative cases for the exon 7 SNP consisted of 18 homozygous cytosine and 11 homozygous guanine alleles. Although the yield of informative cases was not increased by extending the analysis to the two SNP sites (Table 2), assessing both sites provided quality control by independent confirmation of genotyping results (Fig. 2). No discrepancies were found in allelic imbalance analysis for any informative case in this series. Eighteen informative cases were identified using both intron 4 and exon 7 SNP sites.

Of 18 informative cases, 11 (61.1\%) demonstrated allelic imbalance of the hOGG1 gene (Tables 1 and 2). The frequency $(61.1 \%)$ of the hOGG1 allelic loss in HNSCC in this study is similar to that (55\%) obtained by Blons et al (1999) using five microsatellite markers. Allelic loss in our study affected both polymorphic alleles with equal frequency (Table 2).

To define the temporal onset of allelic loss, areas representative of benign hyperplastic squamous mucosa, carcinoma in situ, and invasive SCC were separately microdissected (Fig. 1) for allelic imbalance determination in two cases (Cases 3 and 4 in Table 1). The hOGG1 allelic loss was present in both in situ and invasive SCC components, but not in the adjacent benign hyperplastic squamous mucosa in both cases. In one case (Case 4), multiple samples of histologically benign and hyperplastic squamous mucosa adjacent to the invasive carcinoma were separately microdissected and analyzed for hOGG1 allelic loss. Normal or 

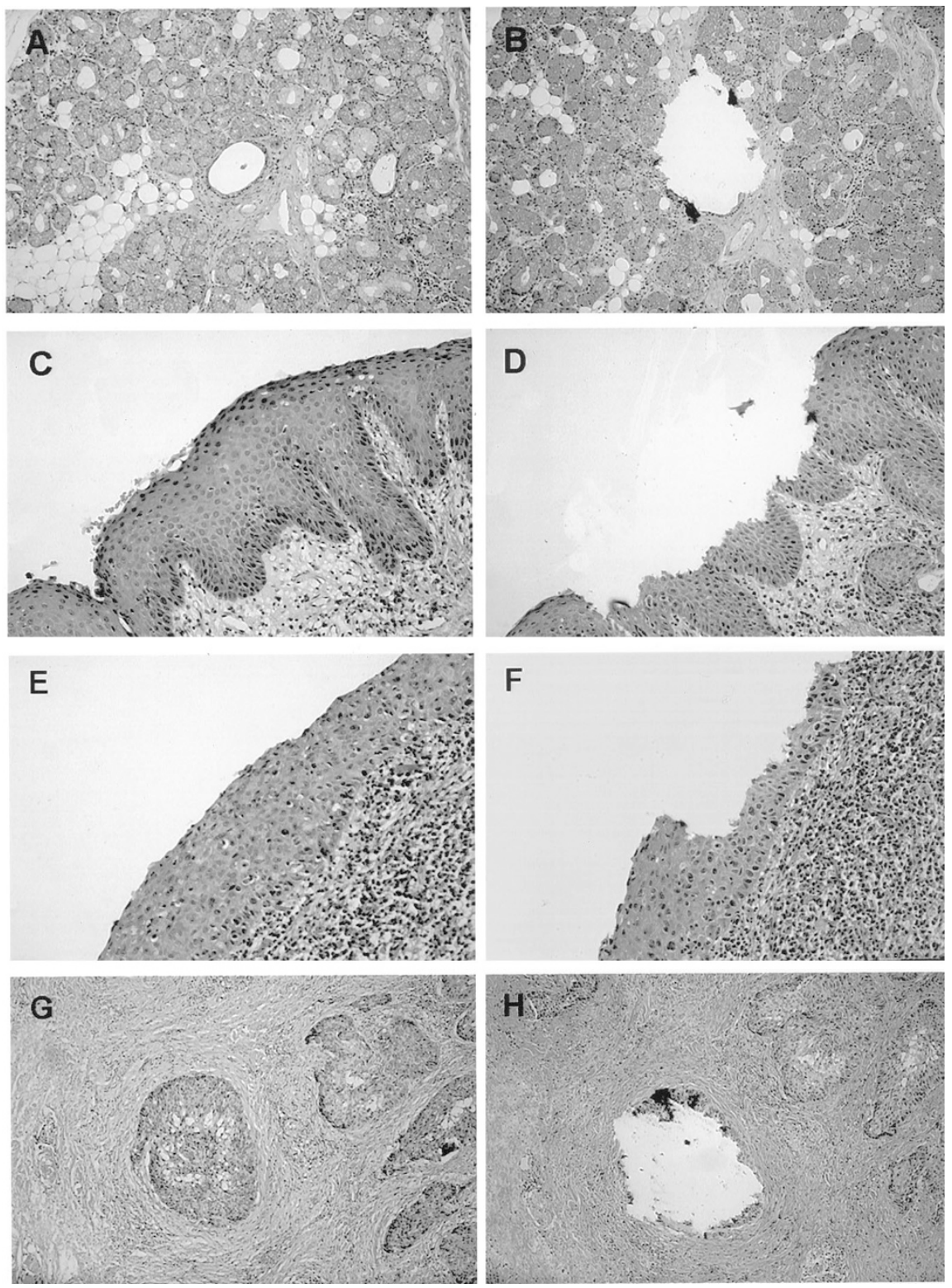

\section{Figure 1.}

Hematoxylin and eosin-stained histopathologic sections consisting of normal minor salivary gland ( $A$ and $B$ ), normal squamous mucosa adjacent to invasive carcinoma ( $C$ and $D)$, carcinoma in situ $(E$ and $F)$, and invasive squamous carcinoma $(G$ and $H)$, before $(A, C, E$, and $G)$ and after $(B, D, F$, and $H)$ microdissection. Under stereomicroscope (SZ-40; Olympus, Tokyo, Japan), the areas of interest can be fairly accurately targeted and removed manually using a pointed surgical blade.

benign hyperplastic squamous mucosa showed no evidence of allelic loss, whereas adjacent invasive tumor displayed apparent hOGG1 allelic loss (Fig. 3).

\section{Immunohistochemical (IHC) Staining for the hOGG1 Protein}

A total of 71 HNSCC cases, including 15 that were used for hOGG1 gene LOH analysis (Cases 4 to 18 in Table 1), were further analyzed with $\mathrm{IHC}$ staining procedure using a polyclonal antibody against the hOGG1 peptide. Three of 18 cases with the hOGG1 $\mathrm{LOH}$ results (Cases 1, 2, and 3 in Table 1) were devoid of a carcinoma component in the tissue blocks due to repeated sectioning and thus were not included in this IHC study. Among these 71 cases used for IHC study,
58 cases contained both normal squamous mucosa and invasive carcinoma, whereas the remaining 13 cases contained only an invasive carcinoma component. Normal squamous mucosa was positively stained in all but two cases, indicative of the presence of endogenous hOGG1 activity. In normal squamous epithelium, the most actively proliferating cells (basal and parabasal, cells) were strongly positive for the hOGG1 protein in the nuclei (Fig. 4, $A$ and $D$ ), whereas the highly differentiated, mitotically inactive superficial keratinocytes were only weakly positive or negative for the hOGG1 protein (Fig. 4, A and D).

In invasive carcinoma, the nuclear staining for the hOGG1 protein was highly heterogeneous with both 

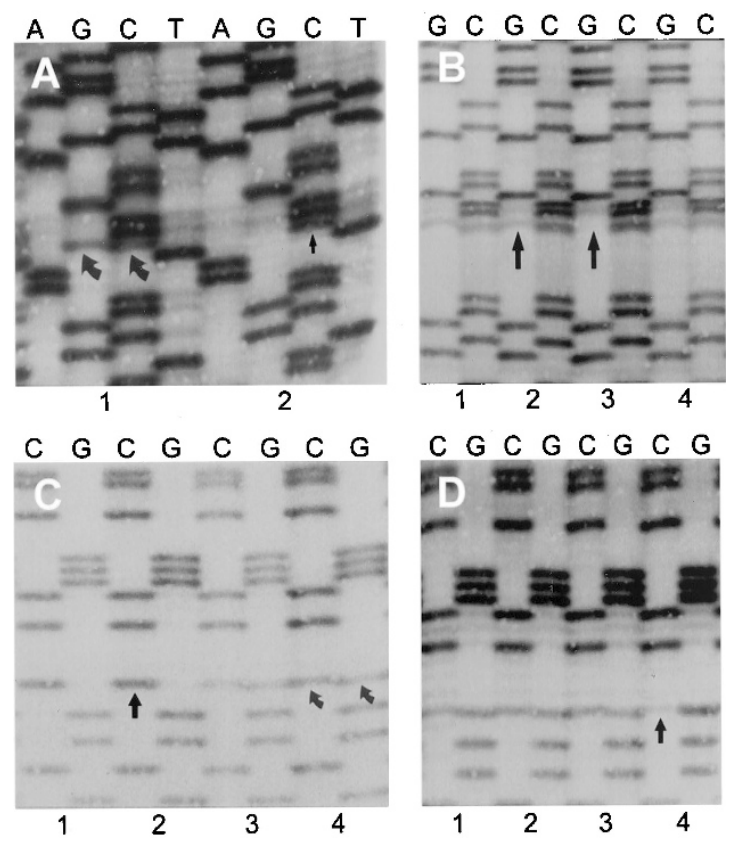

Figure 2.

Microdissection-based allelic imbalance analysis. A, A single nucleotide polymorphism (SNP) site $(G / C)$ in exon 7 is used with DNA from normal minor salivary glands to identify informative cases. DNA sample 1 shows both polymorphic bases (curved arrows) and is, therefore, informative. DNA sample 2 is noninformative at this site (straight arrow). B, Corresponding microdissected tumor samples from the exon 7-informative cases are evaluated for allelic imbalance. Note the absence or significantly diminished intensity of one of the two allelic bands (arrows) in DNA samples 2 and 3. This finding indicates the presence of allelic imbalance. C, An SNP site $(G / C)$ in intron 4 is used with DNA from normal minor salivary glands to identify informative cases. DNA sample 4 is an example of an informative case in which both polymorphic bases are clearly visible (curved arrows). DNA sample 2 represents a noninformative case (straight arrow). D, Corresponding microdissected tumor samples from the intron 4 informative cases are evaluated for allelic imbalance. Note the markedly decreased band intensity in one of the two allelic bands (arrow) in DNA sample 4. This finding indicates the presence of allelic imbalance. (Note: DNA samples are identified by number only for the convenience of interpretation. The numbered samples are not the same samples in Panels A, B, C, and D.)

positively and negatively stained tumor cells in almost all cases, although in different proportions. As shown in Figure 4, Panels $A$ to $C$ were derived from a case that was very strongly positive, whereas Panels $D$ to $F$ were from a case that was negative for the hOGG1 protein. In both cases, the surface squamous epithelia were strongly positive for the hOGG1 protein, particularly in the basal and parabasal cells (A and D), as well as early invasive carcinoma cells (A). Both positive and negative cases displayed a heterogeneous pattern of staining in most areas of the tissue sections ( $B$ and E). In the positive case, most tumor cells were positive for the hOGG1 protein, except for a few well-differentiated keratinocytes in the center of the squamous pearl (B). By comparison, the carcinoma cells in the negative case were mostly negative for the hOGG1 protein, except for some scattered positive cells at the periphery of the tumor lobules. Focally, a homogenous staining pattern was also recognized $(\mathrm{C}$ and F). In these areas, the tumor cells in the positive case were all positive (C), whereas those in the negative case were all negative $(F)$ for the hOGG1 protein.
The stromal spindled cells were generally negative for the DNA repair protein $(B, C, E$, and $F)$.

Of 71 cases that were subjected to IHC study, 27 $(38 \%)$ cases were negative (6) or mostly negative (21); $8(11 \%)$ cases were weakly (2) or moderately (6) positive; and 36 (51\%) cases were strongly (18) or very strongly (18) positive for the hOGG1 protein. Of the 27 cases in which the invasive carcinoma cells were negative or mostly negative for the hOGG1 protein, three contained carcinoma in situ component, and the in situ components in all three cases were strongly positive for the hOGG1 protein.

In 5 of $8(63 \%)$ cases that showed $\mathrm{LOH}$ in the hOGG1 gene (Table 1), the carcinoma cells were negative or mostly negative for the hOGG1 protein, with the remaining three $\mathrm{LOH}+$ cases being moderate to strongly positive for the protein (Cases 5, 6, and 16 in Table 1). By contrast, in 6 of $7(86 \%)$ cases that showed no evidence of the hOGG1 allelic loss (Table 1), carcinoma cells were strongly or very strongly positive for the hOGG1 protein, with the remaining case being moderately positive (Case 9 in Table 1). Unfortunately, in two cases (Cases 3 and 4 in Table 1), in which the in situ carcinoma component showed evidence of hOGG1 allelic loss, the in situ components were lost due to repeated sectioning, precluding further IHC study.

\section{Discussion}

The presence or absence of hOGG1 allelic loss was determined in this study by microdissection of formalin-fixed, paraffin-embedded tissue sections. This method could easily be applied to clinical specimens processed in the course of routine pathology practice. Single recut histologic sections provided sufficient microdissected tissue not only for analysis of two single nucleotide polymorphisms in the hOGG1 gene, but for up to 10 additional genotyping reactions. The approach outlined in this report therefore provides a simple means to evaluate the interaction between multiple cancer genes.

Due to the need to sample minute amounts of tissue, PCR played an essential role in amplifying sufficient DNA for genotyping reactions. Determination of allelic loss or gene gain can be achieved by a variety of techniques; however, many techniques are unsuitable because they require relatively large amounts of fresh tissue. The only limiting factor in the allelic imbalance approach described herein is the requirement for accurate sequence information concerning the precise location of the intragenic SNP site and genomic sequence data sufficient to design amplification primers. In this way, the methods used can be easily applied to other genes susceptible to allelic deletion.

An important limiting factor in the analysis is the realization that only a proportion of test subjects was found to be informative. This limited allelic imbalance determination in only a subset $(40 \%)$ of 45 cases. The availability of a second SNP within the hOGG1 gene was not capable of increasing the yield of informative 
Table 1. hOGG1 Allelic Imbalance and IHC Staining: Results of 18 Informative HNSCC Cases

\begin{tabular}{rllllll}
\hline Case & Liagnosis & Location & $\begin{array}{c}\text { Clinical } \\
\text { stage }\end{array}$ & $\begin{array}{c}\text { Informative } \\
\text { Ex7/In4 }\end{array}$ & $\begin{array}{c}\text { IHC } \\
\text { Staining }\end{array}$ \\
\hline 1 & SCC & Tongue & IV & Yes/Yes & Yes & NA \\
2 & SCC & Tongue & IV & Yes/Yes & Yes & NA \\
3 & SCIS & Buccal & No/Yes & Yes & NA \\
4 & SCC & Tongue & IV & Yes/Yes & Yes & - \\
5 & VSCC & Alveolar & II & Yes/Yes & Yes & ++ \\
6 & SCC & Tongue & III & Yes/Yes & Yes & +++ \\
7 & SCC & Tongue & IV & Yes/Yes & No & +++ \\
8 & SCC & Buccal & II & Yes/No & No & ++++ \\
9 & SCC & Tongue & III & Yes/Yes & No & ++ \\
10 & SCC & Tongue & III & Yes/Yes & No & ++++ \\
11 & SCC & Tongue & III & Yes/Yes & No & +++ \\
12 & BSCC & Larynx & III & Yes/Yes & Yes & - \\
13 & BSCC & Larynx & IV & No/Yes & No & ++++ \\
14 & BSCC & Larynx & IV & Yes/Yes & Yes & - \\
15 & BSCC & Tongue & IV & Yes/Yes & Yes & $-/+$ \\
16 & BSCC & Larynx & IV & Yes/No & Yes & +++ \\
17 & BSCC & Tongue & IV & Yes/Yes & No & +++ \\
18 & BSCC & Larynx & IV & Yes/Yes & Yes & $-/+$
\end{tabular}

hOGG1, human 8-oxoguanine DNA glycosylase; HNSCC, head and neck squamous cell carcinoma; SCC, conventional squamous cell carcinoma; SCIS, squamous cell carcinoma in situ; VSCC, verrucous squamous cell carcinoma; BSCC, basaloid squamous cell carcinoma; Ex7, exon 7; In4, intron 4; LOH, Ioss of heterozygosity; IHC, immunohistochemical; NA, not available.

Table 2. h0GG1 Allelic Imbalance in HNSCC

\begin{tabular}{cccccc}
\hline Cancer type & $\begin{array}{c}\text { Total cases } \\
\text { examined }\end{array}$ & $\begin{array}{c}\text { Intron 4 SNP } \\
\text { informative }\end{array}$ & $\begin{array}{c}\text { Exon 7 SNP } \\
\text { informative }\end{array}$ & $\begin{array}{c}\text { Total informative } \\
\text { cases }\end{array}$ & $\begin{array}{c}\text { Cases with allelic } \\
\text { imbalance } \\
(+\mathrm{LOH})\end{array}$ \\
\hline SCC & 28 & 9 & 9 & 10 & $5(50 \%)$ \\
BSCC & 16 & 6 & 6 & 7 & $5(71 \%)$ \\
VSCC & 1 & 1 & 1 & 1 & $1(100 \%)$ \\
Total & 45 & $16(36 \%)$ & $16(36 \%)$ & $18(40 \%)$ & $11(61 \%)$ \\
\hline
\end{tabular}

SNP, single nucleotide polymorphism.

patients dramatically because the two sites were shown to be in linkage disequilibrium (Table 2). This result is consistent with that obtained by Kohno et al (1998). In that study, 19 of 45 (42\%) cases of primary lung tumors were determined to be informative using a SNP site in exon 7 of hOGG1 gene. When four different SNP sites at 5'-noncoding region, exon 2, intron 4, and exon 7, were used for analysis, the total number of informative cases was only slightly increased to 23 (51\%) (Kohno et al, 1998). Nevertheless, extension of the analysis to the second site in this study provided an independent means for molecular confirmation of the presence or absence of allelic loss. The approach provided an excellent means to insert quality control in the genotyping.

In two cases (Cases 3 and 4 in Table 1), the hOGG1 allelic loss was detected in in situ carcinoma cells but not in the adjacent normal or benign hyperplastic squamous mucosa. It has been demonstrated that the allelic loss of chromosome 3p where the hOGG1 gene resides was acquired as early as in the dysplastic cells (Califano et al, 1996). Although the hOGG1 allelic loss appeared to be acquired in early noninvasive SCC in both of our cases, this finding needs to be confirmed in a larger series of cases that contain dysplastic squamous cells and/or in situ carcinoma.

We observe a high frequency of hOGG1 LOH in a series of HNSCC patients. However, this result, by itself, does not represent compelling supportive evidence for the roles of the hOGG1 gene in head and neck carcinogenesis, nor does it support possible loss of hOGG1 function in those HNSCC cases with evidence of hOGG1 LOH, because the other allele of the gene may remain functional. To characterize hOGG1 gene function in HNSCC, we decided to examine the protein expression patterns by $\mathrm{IHC}$ staining procedure using a commercially available anti-hOGG1 antibody. Although the staining pattern was in general heterogeneous, we were able to show that marked suppression of the hOGG1 gene expression was seen in 27 of $71(38 \%)$ cases of head and neck cancers. In another $8(11 \%)$ cases of head and neck cancer, 30\% to $50 \%$ of the invasive carcinoma cells were negative for the hOGG1 protein expression (see "Results"), indicating a milder degree of functional suppression of the hOGG1 gene. Taking these results all together, we 


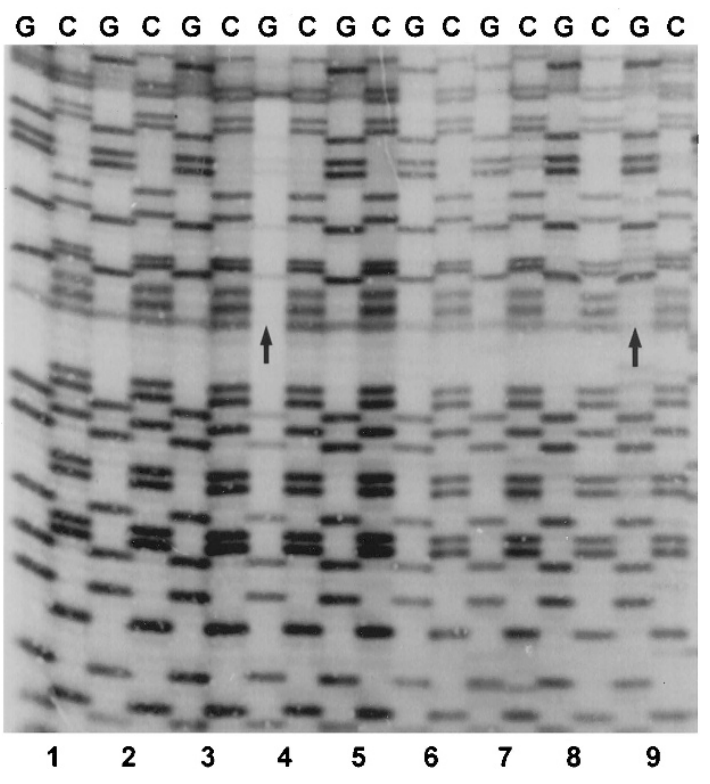

Figure 3.

Loss of heterozygosity ( $\mathrm{LOH}$ ) analysis of normal and neoplastic squamous epithelium using exon 7 SNP. These nine microdissected tissue samples were obtained from a subject (Case 4 in Table 1) manifesting human 8-oxoguanine DNA glycosylase 1 (hOGG1) allelic loss in the tumor. Samples 1, 2, 3, 6, 7, and 8 were obtained from histologically benign but hyperplastic squamous mucosa. These samples show no evidence of allelic loss. Samples 4 and 9 are from the tumor and confirm allelic imbalance. Sample 5 is taken from normal-appearing minor salivary gland and serves as an internal negative control.

concluded that hOGG1 gene suppression in HNSCC is a fairly common event.

Among 71 cases of head and neck cancer studied for hOGG1 gene expression by IHC staining, 15 were previously analyzed for the hOGG1 gene allelic imbalance. Results on these 15 cases indicated that the majority of the invasive carcinoma cells were negative for the hOGG1 protein in 5 of $8(63 \%)$ cases that demonstrated allelic loss of the hOGG1 gene, whereas most of the tumor cells were positive for the DNA repair protein in 6 of $7(86 \%)$ cases that showed no evidence of hOGG1 allelic loss. Thus, in our small series of cases, there was at least some degree of correlation between hOGG1 gene allelic loss and functional suppression. We should, however, caution that the relationship between these two is complex and further studies, such as molecular analysis of the remaining allele for mutation or promoter methylation in a larger series of cases, need to be pursued to establish any relationship, if at all, between hOGG1 allelic deletion and gene function.

The lack of correlation between hOGG1 gene allelic deletion and functional suppression demonstrated by IHC staining in three cases (Cases 5, 6, and 16 of Table 1) may reflect the fact that in some invasive oral SCC, hOGG1 allelic loss may be a secondary event caused by deletion of an adjacent unknown tumor suppressor gene.

The causes of the apparent suppression of hOGG1 gene expression in some HNSCC remain to be determined. Both genetic (somatic mutations of the coding sequence of the gene) and epigenetic (promoter hypermethylation) factors should be examined in this regard.

In a report by Blons et al (1999), the investigators failed to find any identifiable mutations in the hOGG1 gene-coding sequence in HNSCC cases with evidence of hOGG1 allelic loss. This result, in combination with those by others (Chevillard et al, 1998; Kohno et al, 1998; Shinmura et al, 1998), indicates that somatic mutations of the hOGG1 gene appear to be very rare events in tumors of the upper aerodigestive tract and that promoter hypermethylation should be explored as a possible epigenetic mechanism for gene inactivation.

In human cancers, hypermethylation has been well documented in the $\mathrm{CpG}$ islands of the promoter region of a variety of housekeeping genes, resulting in gene inactivation. For example, although germline and/or somatic mutations of a DNA mismatch repair gene, hMLH1, were frequently found in colon cancers from patients with hereditary nonpolyposis colorectal cancer (Bronner et al, 1994; Papadopoulos et al, 1994), somatic mutations of this gene cannot be identified in the majority of the sporadic forms of the tumor (Arzimanoglou et al, 1998; Kok et al, 1997). Yet many of these sporadic colon cancers demonstrated a lack of hMLH1 protein expression and this was the result of epigenetic gene inactivation due to hypermethylation in the CpG islands of the hMLH1 gene promoter (Maekawa et al, 1999; Toyota et al, 1999). Promoter hypermethylation in the $\mathrm{CpG}$ islands has been described in another housekeeping DNA repair gene, $\mathrm{O}^{6}$-methylguanineDNA methyltransferase (MGMT), resulting in gene inactivation in a variety of human cancers (Esteller et al, 1999). In HNSCC, promoter hypermethylation of the MGMT gene occurred in approximately $30 \%$ of the cases in two separate studies (Esteller et al, 1999; Sanchez-Cespedes et al, 2000).

The hOGG1 gene promoter has been characterized and is shown to be devoid of TATA or CAAT boxes, suggesting that hOGG1 is a housekeeping gene (Dh'naut et al, 2000). It has also been shown that the promoter and $5^{\prime}$ sequences of the transcribed region of the hOGG1 gene contain two CpG islands (Dh'naut et al, 2000), and this raises the possibility that promoter hypermethylation of the hOGG1 gene may constitute an important mechanism of gene inactivation. Promoter methylation could be analyzed by methylation-specific PCR, in which DNA will be chemically modified with sodium bisulfite treatment, followed by PCR amplification of the modified DNA (Esteller et al, 1999; Herman et al, 1994).

In summary, we show that LOH and functional suppression of the hOGG1 gene are common in HNSCC. The hOGG1 gene, thus, may represent a highly relevant target in the future studies that aim at elucidating the roles of oxidative DNA damages and DNA repair in head and neck squamous carcinogenesis. 

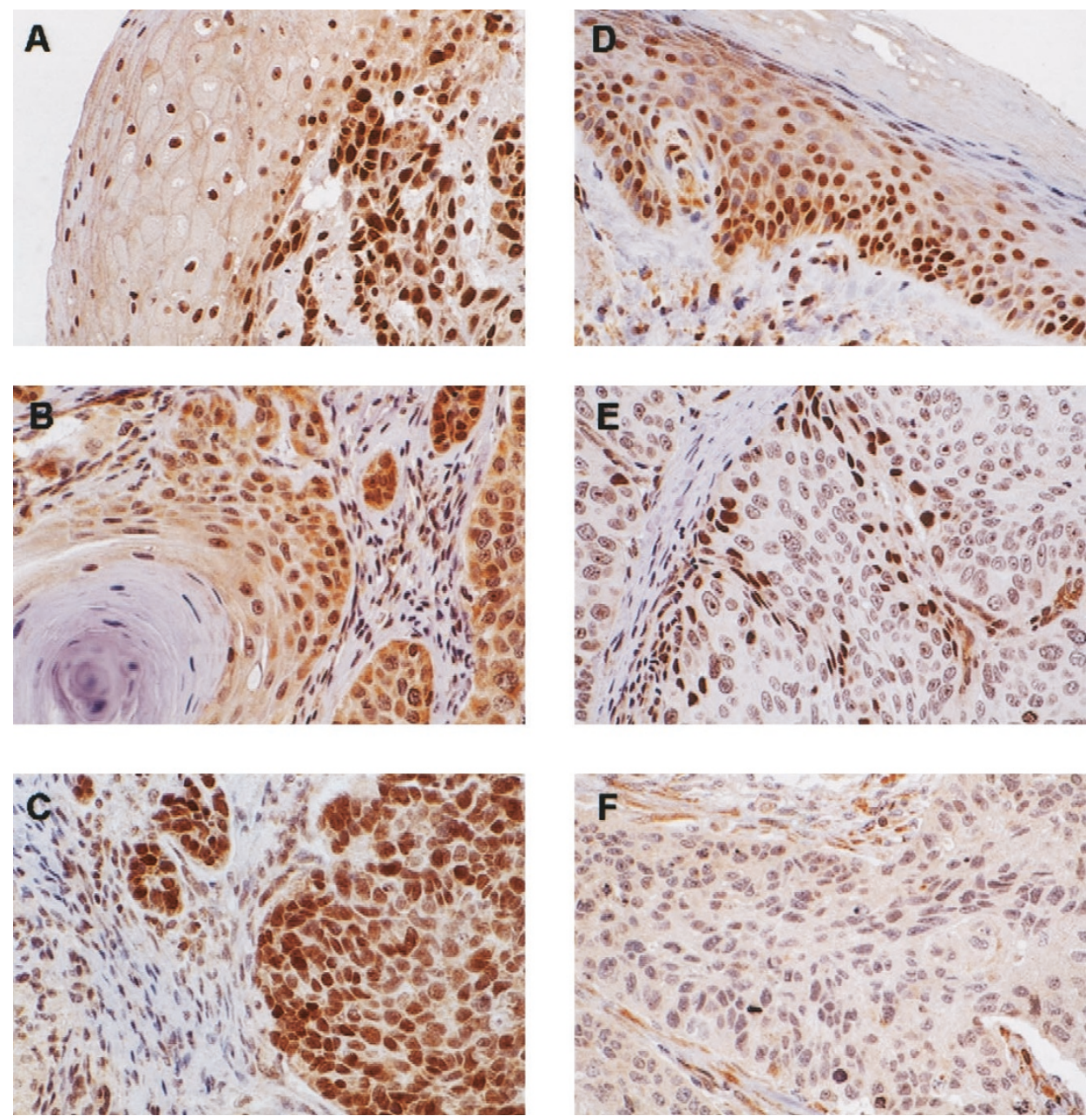

\section{Figure 4.}

Immunohistochemical (IHC) staining for the hOGG1 protein from one case in which a majority of the invasive carcinoma cells are positive (A, B, C) and from another case in which most tumor cells are negative (D, E, F) for the hOGG1 protein. In normal squamous epithelia, intense nuclear immunostaining is seen, particularly in the basal and parabasal cells (A, D). In Panel A, early invasive carcinoma cells are also strongly positive for the hOGG1 protein. The hOGG1 IHC staining pattern is in general heterogenous. Invasive tumor cells in the positive case are mostly positive for the hOGG1 protein except for a few well-differentiated keratinocytes in the center of the squamous pearl (B). By comparison, the carcinoma cells in the negative case were mostly negative except for some positive cells at the periphery of the tumor lobules (E). Focally, a homogeneous staining pattern was also recognized ( $\mathrm{C}$ and $\mathrm{F}$ ). In this area, the tumor cells in the positive case were all positive (C), whereas those in the negative case were all negative $(F)$ for the hOGG1 protein.

\section{Materials and Methods}

\section{Microdissection and DNA Sample Collection}

Twenty-nine samples of oral SCC and 16 samples of tongue and laryngeal basaloid SCC were gathered from the paraffin block archives from 45 patients in the University of Pittsburgh Medical Center, spanning a period from 1997 to 1999 for conventional SCC and from 1991 to 1996 for basaloid SCC. The histology of each case was reviewed and representative tissue sections containing areas of invasive or in situ SCC were selected for microdissection (Fig. 1). Normalappearing salivary gland was microdissected separately to serve as an internal nonneoplastic control (Fig. 1).

Using $4 \mu \mathrm{m}$-thick recut unstained histologic sections, normal and malignant tissue samples were removed under stereomicroscopic observation as previously described (Finkelstein et al, 1996; Papadaki et al, 1996). Sufficient material was collected from a single histologic section to afford replicate analysis of hOGG1 allelic imbalance as described below. Samples were treated with proteinase $\mathrm{K}$ at a final concentration of $100 \mu \mathrm{g} / \mathrm{ml}$ for 2 hours and then boiled for 5 minutes to remove protease activity. To rule out the possibility of allelic dropout as a cause for apparent allelic imbalance in tumor samples (Dreesen et al, 1996; Radinsky et al, 1995), all microdissected normal tissue samples of salivary gland tissue were no larger than corresponding tumor samples from the individual patient (see below).

\section{LOH Analysis}

PCR analysis used two sets of amplification primers encompassing two recently reported single nucleotide polymorphism sites situated in intron 4 and exon 7 of the hOGG1 gene (Kohno et al, 1998). Amplification primers 
for intron 4 were 5'-GGCCACATGCTGCCCTTC-3', sense and 5'-CAGATGCAGTCAGCCACC-3', antisense. Amplification primers for exon 7 were $5^{\prime}-$ CAGACTCCACCCTCCTAC-3', sense and 5'-CGCTTTGCTGGTGGCTCC- $3^{\prime}$, antisense. PCR products were subjected to electrophoresis in $4 \%$ agarose and the ethidium bromide stained bands were excised and then isolated with glassmilk. Allelic imbalance analysis was performed as previously described, based on relative DNA sequencing band intensity between normal and tumor in informative subjects (Finkelstein et al, 1999). DNA sequencing used antisense PCR primers for each single nucleotide polymorphism with $\mathrm{P}^{33}$-dATP as the reporter molecule. Sequence analysis was read from autoradiograms of $6 \%$ polyacrylamide gels that had been exposed overnight.

The following criteria were used to select and evaluate patients for allelic imbalance analysis. To be informative, the normal microdissection tissue sample of minor salivary gland or skeletal muscle was required to show both polymorphic deoxynucleotide bands. Each polymorphic band of the normal tissue was required to exhibit approximately $50 \%$ expected band intensity consistent with equal representation of polymorphic alleles. Of the 46 samples of HNSCC in this study, 18 were classified as informative based on these criteria, and these samples were selected for allelic imbalance analysis of the corresponding tumor samples. Alleles were designated in balance (no allelic loss) when tumor samples manifested equal or nearequal intensity of polymorphic bands. Allelic imbalance (allelic loss) was present when polymorphic bands from tumor tissue in informative subjects were unequivocally of asymmetric intensity. The analysis on all informative subjects was confirmed by replicate analysis using new starting microdissection targets. It should be noted that by strictly limiting normal tissue samples of minor salivary gland to a size no larger than that of corresponding tumor samples, allelic dropout could be excluded.

\section{IHC Staining Procedure}

Fifty-six samples of oral SCC were collected from the paraffin block archives in the Department of Pathology, University of Arkansas for Medical Sciences and Central Arkansas Veterans Healthcare System, spanning a period from 1995 to 1999 . The histology of each case was reviewed and representative tissue sections containing areas of normal squamous mucosa and invasive or in situ SCC were selected for IHC staining procedure. In addition, 15 samples of HNSCC, in which the status of hOGG1 allelic imbalance was available, were included in the IHC study.

The polyclonal antibody against the hOGG1 peptide was obtained commercially (Novus Biologicals, Littleton, Colorado; Cat\# NB 100-106). This anti-hOGG1 antibody was raised in rabbit against a synthetic hOGG1 peptide (CTVYRGDKSQASRPT). The antibody was affinity-purified and could recognize a band at 39 kd, representing hOGG1 (Novus Biologicals).
hOGG1 IHC staining is assessed semiquantitatively with less than $10 \%$ positive cells being negative $(-)$; $10 \%$ to $20 \%$ positive cells being mostly negative $(+/-) ; 30 \%$ to $40 \%$ positive cells being weakly positive (+); $50 \%$ to $70 \%$ positive cells being moderately positive $(++) ; 70 \%$ to $90 \%$ being strongly positive $(+++)$ and more than $90 \%$ positive cells being very strongly positive $(++++)$.

Biotin/Stretavitin complex with microwave antigen retrieval method was used for $\mathrm{IHC}$ staining. Histologic sections to be stained were deparaffinized in xylene and rehydrated through a decreasing ethanol gradient. The slides were then washed in $\mathrm{ddH}_{2} \mathrm{O}$ for 10 minutes followed by microwave antigen retrieval in citrate buffer solution ( $\mathrm{pH}$ 6.0; Zymed Laboratories, San Francisco, California) for 40 minutes. After washing in $\times 1$ PBS twice for 10 minutes, the slides were incubated in blocking solution (Zymed) for 30 minutes at room temperature. After tapping off the blocking solution, primary antihOGG1 antibody (rabbit polyclonal) was added at the dilution of $1 / 1,000$ and incubated at $4^{\circ} \mathrm{C}$ overnight, followed by incubation at room temperature for 2 hours. The slides were then washed in $\times 1$ PBS twice each for 10 minutes. Then the slides were incubated in a prediluted solution containing biotinylated antirabbit link immunoglobulins (Histostain-Plus Kit; Zymed) for 1 hour at room temperature. After washing in $\times 1$ PBS twice each for 10 minutes, the slides were covered with a prediluted solution containing horseradish peroxidase conjugated to Stretavitin (Histostain-Plus Kit; Zymed) and incubated for 1 hour at room temperature. The slides are then washed in $\times 1$ PBS twice each for 10 minutes, followed by incubation for 3 minutes at room temperature in a pre-diluted chromogen solution containing 3,3-diaminobenzidine (DAB) (liquid DAB substrate kit; Zymed). Washing the slides in running tap water stopped the reaction. Finally, the slides were counterstained with hematoxylin, followed by dehydration through a gradient of alcohol concentrations, cleared in xylene, and mounted in Permount.

\section{Acknowledgement}

The authors wish to thank the office of Grants and Scientific Publications at the University of Arkansas for Medical Sciences for editing the manuscript.

\section{References}

Arzimanoglou II, Gilbert F, and Barber HR (1998). Microsatellite instability in human solid tumors (Review). Cancer 82:1808-1820.

Blons H, Radicella P, Laccourreye O, Brasnu D, Beaune P, Boiteux S, and Laurent-Puig P (1999). Frequent allelic loss at chromosome $3 p$ distinct from genetic alterations of the 8-oxoguanine DNA glycosylase 1 gene in head and neck cancer. Mol Carcinog 26:254-260.

Blot WJ, McLaughlin JK, Winn DM, Austin DF, Greenberg RS, Preston-Martin S, Bernstein L, Schoenberg JB, Stemhagen A, and Fraumeni JFJ (1988). Smoking and drinking in relation to oral and pharyngeal cancer. Cancer Res 48:3282-3287. 
Boiteux S, Gajewski E, Laval J, and Dizdaroglu M (1992). Substrate specificity of the Escherichia coli Fpg protein (formamidopyrimidine-DNA glycosylase): Excision of purine lesions in DNA produced by ionizing radiation or photosensitization. Biochemistry 31:106-110.

Brennan JA, Boyle JO, Koch WM, Goodman SN, Hruban RH, Eby YJ, Couch MJ, Forastiere AA, and Sidransky D (1995). Association between cigarette smoking and mutation of the p53 gene in squamous-cell carcinoma of the head and neck. N Engl J Med 332:712-717.

Bronner CE, Baker SM, Marrison PT, Warren G, Smith LG, Lescoe MK, Kane M, Earabino C, Lipford J, Lindblom A, Tannergard P, Bollag RJ, Godwin AR, Ward DC, Nordenskjold M, Fishel R, Kolodner R, and Liskay RM (1994). Mutation in the DNA mismatch repair gene homologue hMLH1 is associated with hereditary non-polyposis colon cancer. Nature 368:258-261.

Califano J, van der Riet P, Westra W, Nawroz H, Clayman G, Piantadosi S, Corio R, Lee D, Greenberg B, Koch W, and Sidransky D (1996). Genetic progression model for head and neck cancer: Implications for field cancerization. Cancer Res 56:2488-2492.

Centers for Disease Control (1990). Current Trends: Deaths from Oral Cavity and Pharyngeal Cancer-United States, 1987. MMWR Morb Mortal Wkly Rep 39:457-458, 460.

Cheng KC, Cahill DS, Kasai H, Nishimura S, and Loeb LA (1992). 8-Hydroxyguanine, an abundant form of oxidative DNA damage, causes G-T and A-C substitutions. J Biol Chem 267:166-172.

Chevillard S, Radicella JP, Levalois C, Lebeau J, Poupon M, Oudard S, Dutrillaux B, and Boiteux S (1998). Mutations in OGG1, a gene involved in the repair of oxidative DNA damage, are found in human lung and kidney tumors. Oncogene 16:3083-3086.

Cowan JM, Beckett MA, Ahmed-Swan S, and Weichselbaum RR (1992). Cytogenetic evidence of the multistep origin of head and neck squamous cell carcinomas. J Natl Cancer Inst 84:793-797.

Dh'naut A, Boiteux S, and Radicella JP (2000). Characterization of the hOGG1 promoter and its expression during the cell cycle. Mutat Res 461:109-118.

Dizdaroglu M (1991). Chemical detection of free radicalinduced damage to DNA. Free Radic Biol Med 10:225-242.

Dreesen JC, Bras M, Coonen E, Dumoulin JC, Evers JL, and Geraedts JP (1996). Allelic dropout caused by allele-specific amplification failure in single-cell PCR of the cystic fibrosis delta F508 deletion. J Assist Reprod Genet 13:112-114.

Esteller M, Hamilton SR, Burger PC, Baylin SB, and Herman JG (1999). Inactivation of the DNA repair gene O6methylguanine-DNA methyltransferase by promoter hypermethylation is a common event in primary human neoplasia. Cancer Res 59:793-797.

Field JK, Spandidos DA, and Stell PM (1992). Overexpression of the p53 gene in head and neck cancer, linked with heavy smoking and drinking. Lancet 339:502-503.

Finkelstein SD, Hasegawa T, Colby T, and Yousem SA (1999). 11q13 allelic imbalance discriminates pulmonary carcinoids from tumorlets: A microdissection-based genotyping approach useful in clinical practice. Am J Pathol 155:633640.
Finkelstein SD, Przygodzki RM, Pricolo VE, Sakallah SA, Swalsky PA, Bakker A, Lanning R, Bland KI, and Cooper DL (1996). Prediction of biological aggressiveness in colorectal cancer by $\mathrm{p} 53 / \mathrm{K}$-ras-2 topographic genotyping. Mol Diagn 1:5-28.

Franceschi S, Talamini R, Barra S, Baron AE, Negri E, Bidoli E, Serraino D, and La Vecchia C (1990). Smoking and drinking in relation to cancers of the oral cavity, pharynx, larynx, and esophagus in northern Italy. Cancer Res 50: 6502-6507.

Gajewski E, Rao G, Nackerdien Z, and Dizdaroglu M (1990). Modification of DNA bases in mammalian chromatin by radiation-generated free radicals. Biochemistry 29:78767882.

Grandis JR, Melhem MF, Gooding WE, Day R, Holst VA, Wagener MM, Drenning SD, and Tweardy DJ (1998a). Levels of TGFa and EGFR protein in head and neck squamous cell carcinoma and patient survival. J Natl Cancer Inst 90:824-832.

Grandis JR, Tweardy DJ, and Melhem MF (1998b). Asynchronous modulation of transforming growth factor $\alpha$ and epidermal growth factor receptor protein expression in progression of premalignant lesions to head and neck squamous cell carcinoma. Clin Cancer Res 4:13-20.

Heo DS, Snyderman C, Gollin SM, Pan S, Walker E, Deka R, Barnes EL, Johnson JT, Herberman RB, and Whiteside TL (1989). Biology, cytogenetics, and sensitivity to immunological effector cells of new head and neck squamous cell carcinoma lines. Cancer Res 49:5167-5175.

Herman JG, Latif F, Weng Y, Lerman MI, Zbar B, Liu S, Samid D, Duan D, Gnarra JR, Linehan WM, and Baylin SB (1994). Silencing of the VHL tumor-suppressor gene by DNA methylation in renal carcinoma. Proc Natl Acad Sci USA 91:97009704.

Hibi K, Takahashi T, Yamakawa K, Ueda R, Sekido Y, Ariyoshi Y, Suyama M, Takagi H, Nakamura Y, and Takahashi T (1992). Three distinct regions involved in $3 p$ deletion in human lung cancer. Oncogene 7:445-449.

Kohno T, Shinmura K, Tosaka M, Tani M, Kim SH, Nohmi T, Kasai H, and Yokota J (1998). Genetic polymorphisms and alternative splicing of the hOGG1 gene, that is involved in the repair of 8-hydroxyguanine in damaged DNA. Oncogene 16:3219-3225

Kok K, Naylor SL, and Buys CHCM (1997). Deletions of the short arm of chromosome 3 in solid tumors and the search for suppressor genes. Adv Cancer Res 71:27-92.

Maekawa M, Sugano K, Kashiwabara H, Ushiama M, Fujita S, Yoshimori M, and Kakizoe T (1999). DNA methylation analysis using bisulfite treatment and PCR-single-strand conformation polymorphism in colorectal cancer showing microsatellite instability. Biochem Biophy Res Comm 262:671-676.

Maestro R, Gasparotto D, Vukosavljevic T, Barzan L, Sulfaro $S$, and Boiocchi M (1993). Three discrete regions at 3p in head and neck cancers. Cancer Res 53:5775-5779.

Michaels ML, Cruz C, Grollman AP, and Miller JH (1992). Evidence that MutY and MutM combine to prevent mutations by an oxidatively damaged form of guanine DNA. Proc Natl Acad Sci USA 89:7022-7025.

Papadaki H, Kounelis S, Kapadia SB, Bakker A, Swalsky PA, and Finkelstein SD (1996). Relationship of p53 gene alterations with tumor progression and recurrence in olfactory neuroblastoma. Am J Surg Pathol 20:715-721. 
Papadopoulos N, Nicolaides NC, Wei YF, Ruben SM, Carter KC, Rosen CA, and Haseltine WA (1994). Mutation of a MutL homolog in hereditary colon cancer. Science 263:1625-1629.

Radicella JP, Dherin C, Desmaze C, Fox MS, and Boiteux S (1997). Cloning and characterization of hOGG1, a human homolog of the OGG1 gene of Saccharomyces cerevisiae. Proc Natl Acad Sci USA 94:8010-8015.

Radinsky R, Risin S, Fan D, Dong Z, Bielenberg D, and Bucana Cea (1995). Level and function of epidermal growth factor receptor predict the metastatic potential of human colon carcinoma cells. Clin Cancer Res 1:19-31.

Reed AL, Califano J, Cairns P, Westra WH, Jones RM, Koch W, Ahrendt S, Eby Y, Sewell D, Nawroz H, Bartek J, and Sidransky D (1996). High frequency of p16 (CDKN2/MTS-1/ INK4A) inactivation in head and neck squamous cell carcinoma. Cancer Res 56:3630-3633.

Ries LG, Hankey BF, and Edwards BK (1990). Cancer Statistics Review 1973-1987. Bethesda: National Cancer Institute, NIH Publ 90-2789, 51.

Sanchez-Cespedes M, Esteller M, Wu L, Nawroz-Danish H, Yoo GH, Koch WM, Jen J, Herman JG, and Sidransky D (2000). Gene promoter hypermethylation in tumors and serum of head and neck cancer patients. Cancer Res 60:892895.

Saranath D, Chang SE, Bhoite LT, Panchal RG, Kerr IB, Mehta AR, Johnson NW, and Deo MG (1991). High frequency of mutations in codons 12 and 61 of $\mathrm{H}$-ras oncogene in chewing tobacco related human oral carcinoma in India. $\mathrm{Br} \mathrm{J}$ Cancer 63:573-578.
Shinmura K, Kohno T, Kasai H, Koda K, Sugimura $H$, and Yokota J (1998). Infrequent mutations of the hOGG1 gene, that is involved in the expression of 8-hydroxyguanine in damaged DNA, in human gastric cancer. Jpn J Cancer Res 89:825-828.

Toyota M, Ahuja N, Ohe-Toyota M, Herman JG, Baylin SB, and Issa JP (1999). CpG island methylator phenotype in colorectal cancer. Proc Natl Acad Sci USA 96:8681-8686.

Van der Kemp PA, Thomas D, Barbey R, De Oliveira R, and Boiteux S (1996). Cloning and expression in Escherichia coli of the OGG1 gene of Saccharomyces cerevisiae, which codes for a DNA glycosylase that excises 7,8-dihydro-8oxoguanine and 2,6-diamino-4-hydroxy-5-Nmethylformamidopyrimidine. Proc Natl Acad Sci USA 93: 5197-5202.

Vokes EE, Weichselbaum RR, Lippman SM, and Hong WK (1993). Head and neck cancer. N Engl J Med 328:184-194.

Whang-Peng J, Banks-Schlegel SP, and Lee EC (1990). Cytogenetic studies of esophageal carcinoma cell lines. Cancer Genet Cytogenet 45:101-120.

Whang-Peng J, Kao-Shan CS, Lee EC, Bunn PA, Carney DN, Gazdar AF, and Minna JD (1982). Specific chromosomal defect associated with human small-cell lung cancer: Deletion 3p(14-23). Science 215:181-182.

Wu LC, Sloan P, Read AP, Harris R, and Thakker N (1994). Deletion mapping on the short arm of chromosome 3 in squamous cell carcinoma of the oral cavity. Cancer Res 54:6484-6488. 